\title{
Medicines used in pregnancy, childbirth and lactation in a teaching hospital in Lagos, Nigeria
}

\author{
Kazeem A Oshikoya ${ }^{1}$, Ireti O Akionla ${ }^{2}$, Idowu O Senbanjo ${ }^{3}$, Ibrahim A Oreagba ${ }^{4}$, Olayinka O Ogunleye ${ }^{1}$
}

Sri Lanka Journal of Obstetrics and Gynaecology 2012; 34: 84-98

\begin{abstract}
Objective: Pharmacotherapy during pregnancy, childbirth and lactation is very challenging because only a few medicines have been specifically tested for safety and efficacy during pregnancy. This study aimed to evaluate the pattern of medicines prescribed and their risk categories during pregnancy, childbirth and lactation.
\end{abstract}

Methods: We performed a retrospective audit of the case files of registered pregnant women who received antenatal care, delivered their babies, and followed up postnatally for a period of six weeks at the Obstetrics and Gynaecology Department of the Lagos State University Teaching Hospital (LASUTH), between 1st July and 31st December 2007. The demographic and obstetric data of the patients, and the medications prescribed during pregnancy, childbirth and pueperium, were extracted.

Results: Altogether, 1536 (95.8\%) case files were analysed. The median age of the patients was 29 (IQR: 27-32) years; their parity was between 0 and 5 . Most of the patients were $\leq 30$ years old $(64.6 \%)$, nulliparous $(70.3 \%)$, booked in the second trimester $(54.2 \%)$, and had spontaneous vertex delivery $(67.7 \%)$. ATC group B $(1577 ; 48.6 \%)$, followed by ATC group $\mathrm{N}(448 ; 13.8 \%)$, were the most frequently prescribed medicines during pregnancy. Eighteen (39.1\%) different types of medicines

${ }^{1}$ Department of Pharmacology, Lagos Sate University College of Medicine, Ikeja, Lagos, Nigeria.

${ }^{2}$ Department of Obstetrics and Gynaecology, Lagos State University College of Medicine, Ikeja, Lagos, Nigeria.

${ }^{3}$ Paediatrics Department, Lagos State University College of Medicine, Ikeja, Lagos, Nigeria.

${ }^{4}$ Department of Pharmacology, College of Medicine, University of Lagos, Idiaraba, Lagos, Nigeria.

Correspondence: Adeola Oshikoya

E-mail: kazeemoshikoya@ymail.com prescribed during pregnancy were in category $\mathrm{C}$ risk. ATC group G medicines (65.4\%) were considerably prescribed in labour. Excluding haematinics, ATC group J medicines (42.2\%) were often prescribed during lactation.

Conclusions: There was considerable use of medicine during pregnancy, childbirth and lactation in this study. Further studies are recommended to guide prophylactic use of antibiotics after uneventful normal childbirth.

Keywords: medication, risk category, pregnancy, childbirth, lactation

\section{Introduction}

Pharmacotherapy during pregnancy may pose a significant risk to the mother and her unborn baby ${ }^{1}$. The potential teratogenesis of some medicines and the physiologic changes which affect medicine disposition in pregnancy are of great concern to clinicians and other health professionals involved in the treatment of pregnant women ${ }^{2,3}$. Medicines are known to account for $1 \%$ of the possible external aetiological factors of congenital defects ${ }^{4,5}$. Correct and cautious use of medicines in pregnancy is therefore important for safe pharmacotherapy to both the mother and her unborn baby. Medicine prescribing in pregnancy and lactation would therefore require good knowledge of teratogenecity, foetal and neonatal effects that are associated with the medicines under consideration ${ }^{6}$.

Generally, pharmacological treatment should be avoided in pregnancy, unless absolutely necessary, because most drugs are potentially harmful to the foetus. However, treatment becomes inevitable when a woman with a chronic medical condition becomes pregnant $^{7}$. The chronic medical conditions such as hypertension, diabetes mellitus, epilepsy, bronchial asthma and sickle cell anaemia will require ongoing or episodic treatments ${ }^{8}$. In addition, there are multiple medical conditions, some of which directly result from the pregnancy or are worsened by it, that may require pharmacological therapy ${ }^{9}$. Also, acute medical problems such as malaria, urinary tract infection, gastrointestinal disorders, migraine and upper 
respiratory tract infection can develop during pregnancy and require pharmacotherapy ${ }^{7}$. Failure to manage these acute and chronic medical conditions may adversely affect the health of both the mother and her unborn baby ${ }^{10}$.

Pregnant women are generally excluded, for ethical reasons, from randomised clinical trials in drug development ${ }^{11}$. This has left questions about the safety of new medications on the developing foetus unanswered, upon drug approval and marketing. Despite the unanswered safety questions, women may intentionally or inadvertently be exposed to various prescription and non-prescription medicines before and during pregnancy. Post marketing surveillance studies have revealed association between many commonly used medicines and various birth defects ${ }^{12-1}$. In addition to safety issue problems, there is paucity of information to guide clinicians on the best dose of a particular medicine to recommend for pregnant women since changes in the body physiology during pregnancy have the potential to require that doses be modified. Despite the numerous knowledge gaps on safety and effectiveness of medications in pregnancy, clinicians prescribe multiple medicines for pregnant women ${ }^{15-17}$ and surprisingly, these women also practice polypharmacy $^{18}$.

Aspirin and other non-steroidal anti-inflammatory drugs (NSAIDs) are non-prescribed or overthe-counter medicines that are often used in pregnancy ${ }^{19}$. The use of aspirin and other salicylates in the last trimester can affect the foetal cardiovascular system. It can also cause alterations in maternal and foetal haemostasis mechanisms, decreased birth weight, and increased perinatal mortality ${ }^{20}$. The use of aspirin a week before or during labour and delivery has been associated with postpartum haemorrhage $\mathrm{e}^{19}$. Its inhibitory effect, as well as those of the NSAIDs, on prostaglandin may also prolong labour and gestation $^{20}$.

Breast-feeding is beneficial for the health of a mother and her child ${ }^{21}$. However, many medicines can be transferred into breast milk causing the risk of breast-feeding to exceed its benefit to the infant, mother, or both ${ }^{22,23}$. Although, a study had reported that the majority of prescription and non-prescription medicines are not found in breast milk after ingestion ${ }^{24}$, there is limited evidence-based data regarding the actual safety of many of these medicines, this therefore calls for caution on medicine use during lactation ${ }^{25}$. Aspirin, magnesium salicylate, and bismuth salicylate are excreted into the breast milk and can induce Reye's syndrome $^{24}$.
Aspirin in the breast milk may cause neonatal rashes, platelet abnormalities and bleeding ${ }^{20}$. Antihistamines, including clestamine and diphenhydramine, can pass into the breast milk and produce drowsiness and irritability in the breast-fed infant ${ }^{24}$.

Although many studies around the world have reported increased use of prescription and nonprescription medicines during pregnancy, there is little information on the extent of medicine use during labour and lactation. The two published studies in Nigeria are based on data that were collected during pregnancy and involved pregnant women from Benin City in the South-Eastern Nigeria ${ }^{15,26}$; for these reasons, existing data may not be generalised to pregnant women in Nigeria.

This study aimed to assess the medicines prescribed to pregnant women during antenatal care, childbirth and lactation at a teaching hospital in Lagos, Nigeria. In addition, the study assessed the risk associated with the medicines according to the United States Food and Drug Administration (US-FDA) pregnancy risk classification ${ }^{27}$ and the World Health Organization (WHO) risk classification during breast feeding ${ }^{28}$.

\section{Methods}

This retrospective descriptive study was conducted at The Lagos State University College of Medicine (LASUTH) in Nigeria and involved registered pregnant women (booked cases) who received antenatal care, delivered their babies, and followed up postnatally for a period of six weeks (pueperium period) at the Obstetrics and Gynaecology Department (Ayinke House) of LASUTH. The study involved patients who were seen over a 6 month period, between 1st July and 31st December 2007. Patients who were HIV infected, had incomplete or unavailable medical records for review, and those who were non-ambulatory on or before two weeks of parturition, were excluded from the study.

An average of 680 pregnant women per month is newly registered for antenatal and received antenatal care at the antenatal clinic of the hospital. Medicines prescribed to the patients during antenatal visits are purchased from either the hospital or community pharmacy.

Eligible cases were identified through the main obstetric register obtained from the medical record of the antenatal clinic. A trained research nurse reviewed 
each case file, and using a form purposely designed for the study, extracted data on age and parity of the patient, and gestational age at booking; medical or gynaecological conditions in the pregnancy; mode of delivery; birth age of the baby; and medications (excluding vaccinations) prescribed during pregnancy, childbirth and pueperium. The lead researcher (KAO) corroborated the information extracted by the research nurse by further reviewing each case file. Where there was a disagreement, the opinion of the second researcher (IOA) superseded.

For ease of data comparison, patients' age were classified as $\leq 30$ years or $>30$ years, a method that was adopted from previous studies on drug utilization during pregnancy ${ }^{17}$. The medicines prescribed during pregnancy, child birth and pueperium are classified according to the World Health Organisation's Anatomical Therapeutic Chemical (WHOATC) classification system ${ }^{29}$. The risk associated with the medicines prescribed in pregnancy was according to the US-FDA risk classification (Category A: controlled studies in pregnant women fail to demonstrate a risk to the foetus in the first trimester with no evidence of risk in later trimesters. The possibility of harm appears remote; Category B: presumed safety based on animal studies, with no controlled studies in pregnant women, or animal studies have shown an adverse effect that was not confirmed in controlled studies in women in the first trimester and there is no evidence of a risk in later trimesters; Category C: Studies in women and animals are not available or studies in animals have revealed adverse effects on the foetus and there are no controlled studies in women. Drugs should be given only if the potential benefits justify the potential risk to the foetus; Category D: There is positive evidence of human foetal risk (unsafe), however in some cases such as a life-threatening illness the potential risk may be justified if there are no other alternatives, and Category X: Highly unsafe: risk of use outweighs any potential benefit. Drugs in this category are contraindicated in women who are or may become pregnant $)^{27}$. Medicines were assigned into a risk group using Physicians' Desk Reference ${ }^{30}$, Pregnancy and Lactation Reference books ${ }^{31,32}$, and online search from the database of safefetus. $\mathrm{com}^{33}$.

The safety, to the mother and the baby, of medicines prescribed during child birth and pueperium was according to the WHO classification system (Category 1: compatible with breastfeeding, no known or theoretical contraindications for their use, safe for the mother to take the medicine while breast- feeding; Category 2: compatible with breastfeeding but monitor infant for side-effects, theoretically, medicine could cause side-effects in the infant but have either not been observed to do so or have occasionally caused mild side-effects; Category 3: avoid if possible, however, if used, monitor infant for side effects. Medicines reported to cause side-effects in the infant but are used if the need is really essential for the mother and no safer alternative is available; Category 4: avoid if possible as it may inhibit lactation, medicine may reduce breast milk production; and Category 5: avoid completely, medicine has dangerous side-effects on the baby. They should not be given to the mothers while breastfeeding ${ }^{28}$.

\section{Ethical issues and statistical analyses}

The study protocol was approved by the ethics committee of LASUTH. All data from the medical records were coded and results presented as median and inter-quartile range (IQR), mean with standard deviation (mean \pm S.D), and frequency distribution with percentage. Statistical analysis of the results was performed using the Statistical Package for the Social Sciences (SPSS), version ${ }^{16}$. Comparisons between age of the patient ( $\leq 30$ years or $>30$ years) and parity or gestational age at booking (first, second or third trimester), with regards to continuous data, was made using the Pearson chi-squared test at a significance level of $\mathrm{P}<0.05$. The student paired $\mathrm{t}$-test was used to compare the proportion of patients with medical conditions who were $\leq 30$ years or $>30$ years.

\section{Results}

\section{Demographics and obstetric history of the patients}

We identified a total of 1604 potential cases. Seventy two cases were excluded because the patients were HIV-infected (24) and had incomplete data (48). Altogether, 1536 (95.8\%) case files were analysed in this study. The median age of the patients was 29 (IQR: 27-32) years; their mean parity was $0.58 \pm 1.03$ (range $0-5)$. The majority of the patients $(992 ; 64.6 \%)$ were $\leq 30$ years old, nulliparous (1080; 70.3\%), booked in the second trimester $(832 ; 54.2 \%)$, and delivered by spontaneous vertex delivery (1040;67.7\%). The deliveries were term $(1504 ; 97.9 \%)$, preterm $(25 ; 1.6 \%)$, and post term $(7 ; 0.5 \%)$. Among the 496 patients who delivered by surgery, 344 (69.4\%) had emergency caesarean section; the rest had elective surgeries. Forty eight $(4.6 \%)$ of those who had spontaneous vertex delivery were assisted. There was a significant association between the age $\leq 30$ years or $>30$ years) and parity of the patients $(\mathrm{P}<0.01)$. Most of the younger pregnant women $(848 ; 55.2 \%)$ were nulliparous. 
Contrarily, there was no significant association between the age ( $\leq 30$ years or $>30$ years) and time of booking of the patients $(\mathrm{P}=0.382)$; however, more young women booked for antenatal care in the second $(552 ; 35.9 \%)$, third $(296 ; 19.3 \%)$ and first $(144 ; 9.4 \%)$ trimesters than older women.

\section{Medicines prescribed during pregnancy}

All the patients had haematinics (iron, vitamin B12 and folic acid) throughout the pregnancy, except eight patients who suffered sickle cell anaemia and had only folic acid and vitamin B12. Another 56 patients who suffered nausea and vomiting were not prescribed haematinics until their condition was stable. Since haematinics were prescribed at every stage of the pregnancy, repeated prescriptions were counted as one. Excluding haematinics, women without any medical or gynaecological problem in pregnancy (963; $62.7 \%$ ) encountered a range of $0-3$ medicines. The majority of these patients $(827 ; 85.9 \%)$ were prescribed no medicine. Others had calcium lactate, ascorbic acid and paracetamol, either alone or in combinations.

Five hundred and seventy three (37.3\%) patients had at least one medical or gynaecological condition in pregnancy, 57 (9.9\%) of these patients had 2-3 medical or gynaecological conditions. In order to avoid overestimation of the number of different medicine exposures in pregnant women with chronic medical or gynaecological conditions, multiple prescriptions for the same medicine (antihypertensives, insulin, salbutamol, and others) were counted once. Table 1 shows the various medical and gynaecological conditions observed in the patients according to their ages and medicines prescribed. The proportion of women afflicted with medical and gynaecological conditions who were $\leq 30$ years old were significantly higher than those who were $>30$ years old $(\mathrm{P}=0.018)$. Malaria fever $(128 ; 22.3 \%)$ afflicted the women most, followed by upper respiratory tract infection (82; $14.3 \%)$, and nausea and vomiting (56;9.8\%). Excluding haematinics, a total of 3245 medicines, comprising of 40 different types, were prescribed to all the women throughout the pregnancy (Table 2). These women encountered an average of 3 medicines (range 2-6) per clinic visit.

The medicines prescribed in pregnancy are classified in Table 2 according to the WHO Anatomical Therapeutic Chemical system of classification. ATC group B (blood and blood-forming organs) medicines were the mostly prescribed (1577; 48.6\%), followed by ATC group N (nervous system- 448; 13.8\%) and ATC group R (respiratory system- 355; 10.9\%). Table 3 describes the potential risk of the medicines prescribed in pregnancy according to the US-FDA risk classification system. The majority of the medicines prescribed were in category C $(18 ; 39.1 \%)$, followed by category B $(16 ; 34.8 \%)$ and category A $(5 ; 10.9 \%)$ medicines. Six $(13 \%)$ medicines were however prescribed from category D. Category X medicines which are absolutely contraindicated in pregnancy were not prescribed.

\section{Medicines prescribed during childbirth}

The list of medicines prescribed during childbirth is presented in Table 4. Medicines for genitourinary system and sex hormone (ATC group G- 1576; 65.4\%), predominated by oxytocin, was the most frequently prescribed in the third or fourth stage of labour. Pentazocine $(272 ; 11.3 \%)$, promethazine $(240 ; 10.0 \%)$ and butyl scopolamine (176; $7.3 \%)$, administered alone or in combinations, were also prescribed excessively, especially in the early intrapartum phase of labour.

\section{Medicines prescribed during lactation}

A high proportion of the patients (1505; 18.9\%) had haematinics (iron, vitamin B12 and folic acid or vitamin B12 and folic acid) during early lactation. Since haematinics were prescribed throughout the six weeks postnatal period, repeated prescriptions were counted once. Table 5 shows the ATC classification of the medicines prescribed during early lactation (pueperium). Excluding haematinics, anti-infective agents (ATC group J- 3353; 42.2\%) were the most frequently prescribed medicines, followed by nervous system medicines (ATC group N-1714; 21.6\%). Further analysis showed that, after excluding haematinics, metronidazole $(1424 ; 17.9 \%)$, ampicillin/cloxacillin $(1319 ; 16.6 \%)$, and paracetamol $(1089 ; 13.7 \%)$ were the most frequently prescribed medicines during early lactation. After excluding haematinics, a total of 6438 medicines; comprising of 31 different types were prescribed to the 1536 women during early lactation (Table 5). Each woman encountered an average of 6 medicines (range 2-10).

The risk category of the medicines prescribed during lactation is presented in Table 6 according to the WHO classification (28). The majority of the medicines belonged to category $1(13 ; 38.2 \%)$ and category $2(11 ; 32.4 \%)$. Four medicines (ergometrine, ascorbic acid, hydrocortisone, and dexamethasone) were compatible with breast feeding but may inhibit lactation when used repeatedly, produce adverse effect when used in large doses, or have no safety data for prolonged use. 

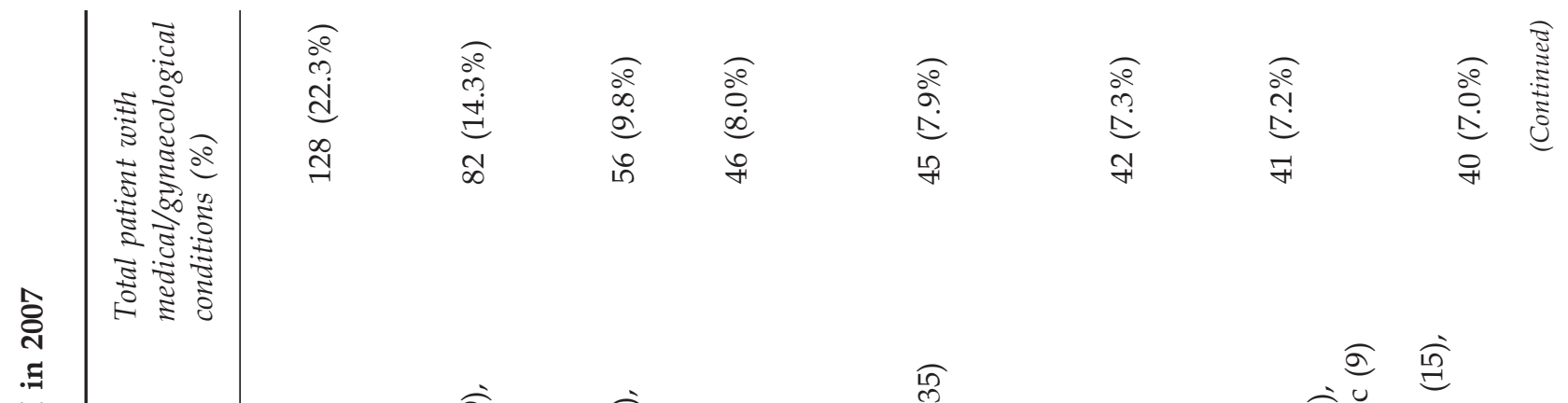

$\stackrel{\overparen{L}}{\stackrel{0}{C}}$

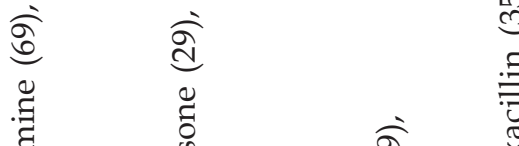

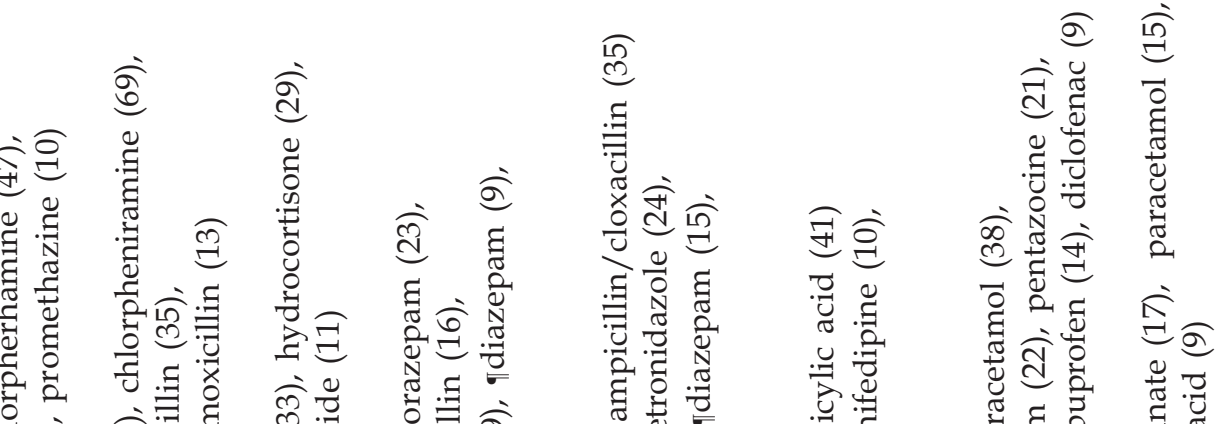

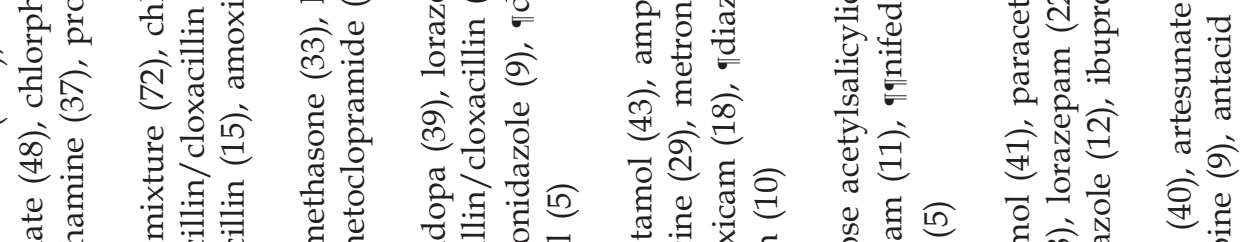

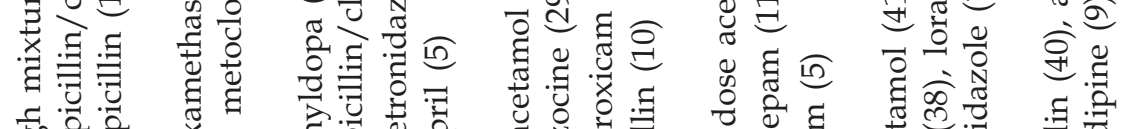

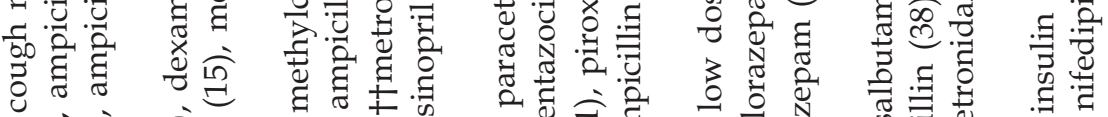
ठ

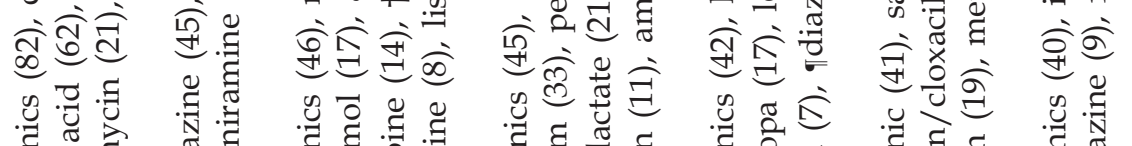

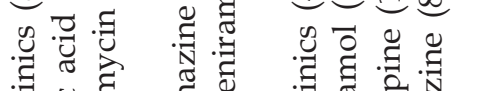

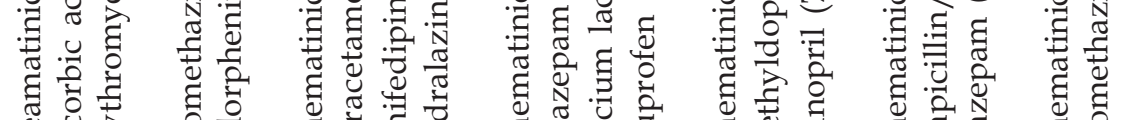

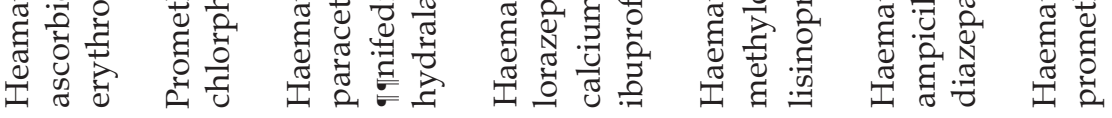

$\bar{z}$

.

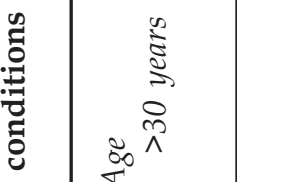

ले $\stackrel{\infty}{\infty} \stackrel{\infty}{\sim}$

$\stackrel{\circ}{\rightarrow}$

$\stackrel{\rightarrow}{\sim} \stackrel{\infty}{\sim}$

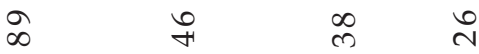

$\stackrel{10}{\infty}$

$\stackrel{\infty}{2}$

i)

$\stackrel{\sim}{\sim}$

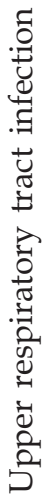

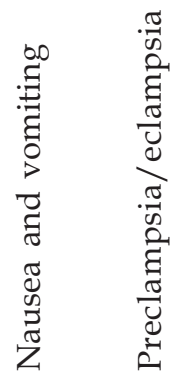

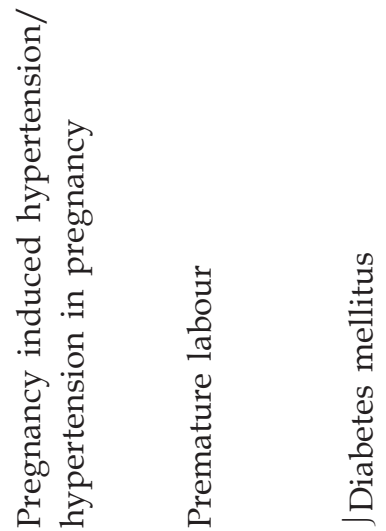




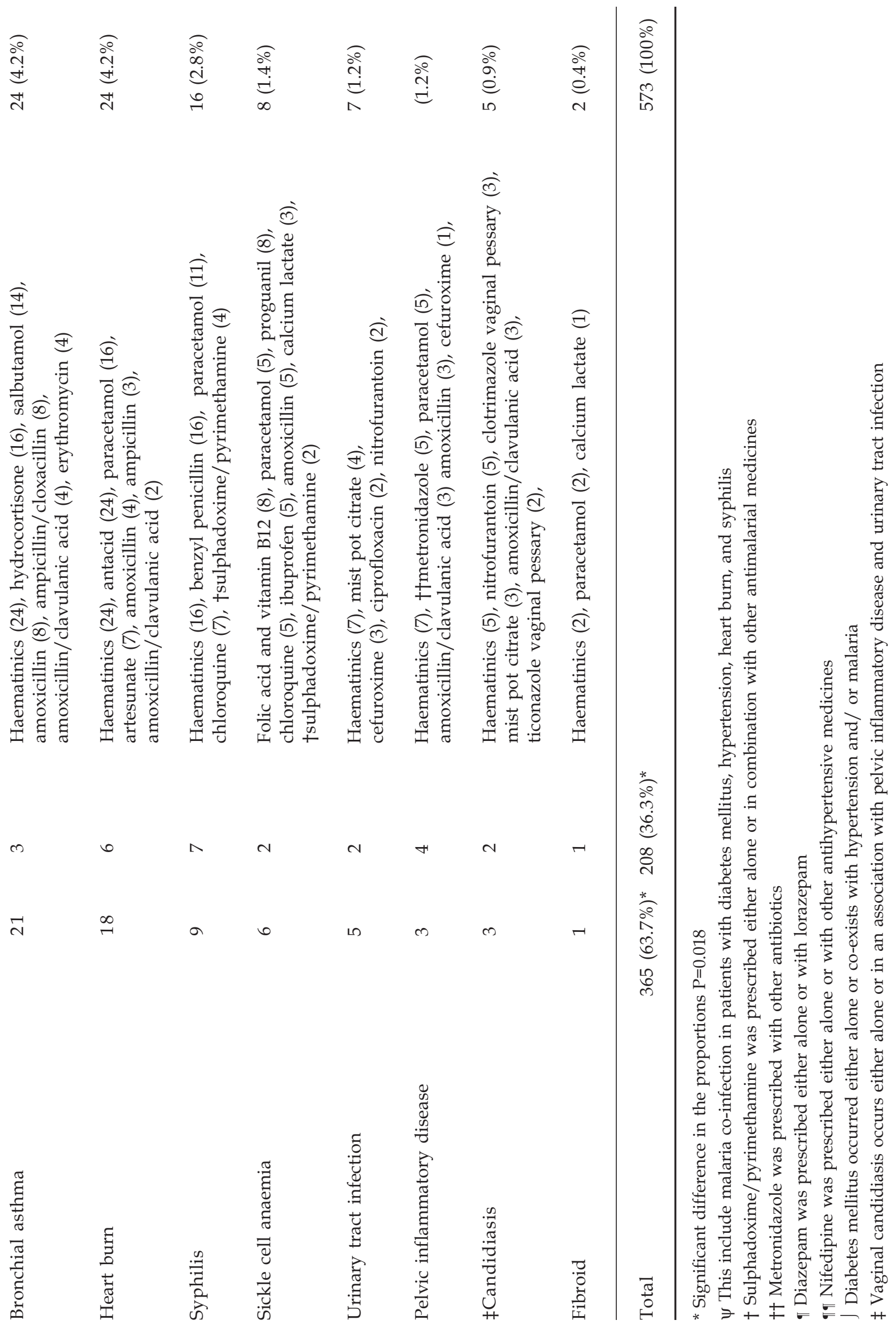


Table 2. Anatomical therapeutic chemical (ATC) classification of medicine prescribed during pregnancy

ATC group and specific medicine Frequency of the specific medicine (n) Total frequency (\%)

A: alimentary tract and metabolism

[A02A] Antacid

[A12AA05] Calcium lactate

[A10AB01] Insulin

[A12BA02] Potassium citrate mixture

[A03FA01] Metoclopramide

[A11GA01] Ascorbic acid

62

B: blood and blood-forming organs

[B01AC06] Acetylsalicylic acid and folic acid

[B03A] Iron, vitamin B12,

[B03B] Vitamin B12and folic acid

64

C: cardiovascular system

[C09AA03] Lisinopril

12

[C02AB] Methyldopa

[C08CA05] Nifedipine

[C02DB01Hydralazine

G: genitourinary system and sex hormones

[G01AF02] Clotrimazole pessary

[G01AG01] Tioconazole pessary

H: systemic hormonal preparations

H02AB09] Hydrocortisone

J: anti-infective for systemic use

[J01CA01] Ampicillin

[J01CA04] Amoxicillin

[J01CE01] Benzyl penicillin

[J01CF01] Ampicillin/cloxacillin

[J01CR02] Amoxicillin/clavulanic acid

[J01DC02] Cefuroxime

[J01FA01] Erythromycin

[J01MA02] Ciprofloxacin

[J01XD01] Metronidazole

[J01XE01] Nitrofurantoin

M: musculoskeletal system

[M01AB05] Diclofenac

[M01AC01] Piroxicam

[M01AE01] Ibuprofen 
$\mathbf{N}$ : nervous system

[N02AD01] Pentazocine 50

[N05BA01] Diazepam

[N05BA06] Lorazepam

[N02BE01] Paracetamol

P: antiparasitic products

[P01BA01] Chloroquine

60

[P01BB02] Proguanil

[P01BD01] Sulphadoxine/pyrimethamine

[P01BE03] Artesunate

R: respiratory system

[R01AD03] Dexamethasone

[R03AC02] Salbutamol 55

[R05DA09] Cough mixture $\quad 72$

[R06AB02] Chlorpheniramine 131

[R06AD02] Promethazine 64

\begin{tabular}{lll}
\hline Total & 3245 & $3245(100 \%)$ \\
\hline
\end{tabular}

Table 3. US-FDA risk category of the medicines prescribed during pregnancy

\begin{tabular}{|c|c|c|}
\hline $\begin{array}{l}\text { Risk } \\
\text { category }\end{array}$ & Medicine & $\begin{array}{l}\text { Type of medicine } \\
\text { per risk category } \\
\quad(\%) n=46\end{array}$ \\
\hline A & $\begin{array}{l}\text { Iron tonic (1472), folic acid (1480), vitamin B12 (1480), } \\
\text { ascorbic acid (62), calcium lactate (25) }\end{array}$ & $5(10.9 \%)$ \\
\hline B & $\begin{array}{l}\text { Paracetamol (261), ampicillin/cloxacillin (132), chlorpheniramine (131), } \\
\text { methydopa (56), metronidazole (50), insulin (40), amoxicillin (33), ampicillin (28), } \\
\text { erythromycin (25), benzyl penicillin (16), amoxicillin/clavulanic acid (12), } \\
\text { metoclopramide (11), ††diclofenac (9), proguanil (8), nitrofurantoin (7), cefuroxime (4) }\end{array}$ & $16(34.8 \%)$ \\
\hline $\mathrm{C}$ & $\begin{array}{l}\text { +Cough mixture (72), promethazine (64), chloroquine (60), salbutamol (55), } \\
\text { pentazocine (50), artesunate (48), hydrocortisone (45), ibuprofen (40), } \\
\text { łsulphadoxine/pyrimethamine (37), nifedipine (33), dexamethasone (33), } \\
\text { antacid (33), piroxicam (18), tlisinopril (12), hydralazine (8), ciprofloxacin (2), } \\
\text { tioconazole pessary (2), clotrimazole pessary (2) }\end{array}$ & $18(39.1 \%)$ \\
\hline $\mathrm{D}$ & $\begin{array}{l}\text { Lorazepam (89), diazepam (48), acetylsalicylic acid (41), } \\
\text { łsulphadoxine/pyrimethamine (37), †lisinopril (12), ††diclofenac (9) }\end{array}$ & $6(13.0 \%)$ \\
\hline$x$ & None & - \\
\hline Uncertain & potassium citrate mixture (7) & $1(2.2 \%)$ \\
\hline
\end{tabular}

+cough mixture contains chorpheniramine, pseudoephedrine, and dextromethorphan or guaifenesin

††diclofenac risk increased from " $\mathrm{C}$ " to " $\mathrm{D}$ " when used in third trimester or near term

†lisinopril risk increased from " $\mathrm{C}$ " to " $\mathrm{D}$ " when used in first trimester

łsulphadoxine/pyrimethamine risk increased from " $\mathrm{C}$ " to “ $\mathrm{D}$ " when used near term 
Table 4. Anatomical therapeutic chemical (ATC) classification of medicines prescribed during childbirth

$\begin{array}{lcc}\text { ATC group and specific medicine } & \text { Frequency of } & \text { Total frequency } \\ (\%)\end{array}$

A: alimentary tract and metabolism

$176(7.3 \%)$

[A03BB01] Butyl scopolamine 176

G: genitourinary system and sex hormones

$1576(65.4 \%)$

[G02AB03] Ergometrine 88

[G02AC01] Oxytocin

1488

J: anti-infective for systemic use

$97(4.0 \%)$

[J01CF01] Ampicillin/cloxacillin

[J01DC02] Cefuroxime

13

[J01GB03] Gentamicin

21

[J01XD01] Metronidazole

30

$\mathbf{N}$ : nervous system

$320(13.3 \%)$

[N02AD01] Pentazocine

272

[N05BA01] Diazepam

48

R: respiratory system

$240(10.0 \%)$

[R06AD02] Promethazine

240

$\begin{array}{lll}\text { Total } & 2409 & 2409(100 \%)\end{array}$

The above list excluded pre-anaesthetic medications used during caesarean section

Table 5. Anatomical therapeutic chemical (ATC) classification of medicine prescribed during lactation

\begin{tabular}{llc}
\hline ATC group and specific medicine & $\begin{array}{c}\text { Frequency } \\
\text { of specific medicine }(n)\end{array}$ & $\begin{array}{c}\text { Total frequency } \\
(\%)\end{array}$ \\
\hline A: alimentary tract and metabolism & $303(3.8 \%)$ \\
[A10AB01] Insulin & 83 & $1536(19.3 \%)$ \\
[A03FA01] Metoclopramide & 262 & \\
[A11GA01] Ascorbic acid & 31 & 1441 \\
B: blood and blood-forming organs & 64 \\
[B01AC06] Acetylsalicylic acid & & \\
[B03A] Iron, vitamin B12, and folic acid & & \\
[B03B] Vitamin B12and folic acid & & \\
\hline
\end{tabular}

(Continued) 
C: cardiovascular system

$282(3.6 \%)$

[C09AA03] Lisinopril

120

[C02AB] Methyldopa

25

[C08CA05] Nifedipine

56

[C03CB01] Furosemide

49

[C02DB01Hydralazine

H: systemic hormonal preparations

$15(0.2 \%)$

[H02AB09] Hydrocortisone

$\mathrm{J}$ : anti-infective for systemic use

15

[J06BB01] Anti-D (rh) immunoglobulin 48

[J01CF01] Ampicillin/cloxacillin 1319

[J01CR02] Amoxicillin/clavulanic acid 9

[J01DC02] Cefuroxime 216

[J01GB03] Gentamicin 320

[J01MA02] Ciprofloxacin 17

[J01XD01] Metronidazole 1424

M: musculoskeletal system

$216(2.7 \%)$

[M01AB05] Diclofenac 95

[M01AC01] Piroxicam 81

[M01AE01] Ibuprofen 40

$\mathrm{N}$ : nervous system

$1714(21.6 \%)$

[N02AD01] Pentazocine 441

[N05BA01] Diazepam 153

N05BA06] Lorazepam 31

[N02BE01] Paracetamol 1089

P: antiparasitic products

$142(1.8 \%)$

[P01BA01] Chloroquine 95

[P01BD01] Sulphadoxine/pyrimethamine 32

[P01BE03] Artesunate 15

R: respiratory system

$382(4.8 \%)$

[R01AD03] Dexamethasone 7

[R03AC02] Salbutamol 7

[R06AB02] Chlorpheniramine 9

[R06AD02] Promethazine 359

$\begin{array}{lll}\text { Total } & 7943 & 7943(100 \%)\end{array}$ 
Table 6. Risk category of medicines prescribed during lactation

\begin{tabular}{|c|c|c|}
\hline Medicine & WHO classification [28] & Comment \\
\hline Ergometrine & 1,4 & $\begin{array}{l}\text { Compatible with breast feeding in single } \\
\text { dose. Repeated doses may inhibit lactation }\end{array}$ \\
\hline Oxytocin & 1 & $\begin{array}{l}\text { Compatible with breast feeding during } \\
\text { short term use }\end{array}$ \\
\hline Anti-D (rh) immunoglobulin & 1 & Compatible with breast feeding \\
\hline Insulin & 1 & Compatible with breast feeding \\
\hline Metoclopramide & 3 & $\begin{array}{l}\text { Avoid if possible. Insufficient data on } \\
\text { prolonged use }\end{array}$ \\
\hline Ascorbic acid & 1,2 & $\begin{array}{l}\text { Compatible with breastfeeding in usual } \\
\text { doses. If large doses are used, monitor for side } \\
\text { effects (hemolysis and jaundice), especially } \\
\text { in premature infants }\end{array}$ \\
\hline
\end{tabular}

Compatible with breastfeeding in occasional doses. Monitor for haemolysis, prolonged bleeding and metabolic acidosis

Haematinics

(Iron, folic acid, vitamin B12)

Lisinopril

Methyldopa

Nifedipine

Furosemide

Hydralazine

Hydrocortisone

Ampicillin/cloxacillin

Amoxicillin/clavulanic acid

Cefuroxime

Gentamicin
1

1

4

1
Compatible with breastfeeding

Compatible with breastfeeding

Compatible with breastfeeding

Compatible with breastfeeding

Avoid if possible. May inhibit lactation

Compatible with breastfeeding

Available data insufficient on prolonged use

Compatible with breastfeeding in single dose. No available data about prolonged use

Compatible with breast feeding

Compatible with breast feeding

Compatible with breast feeding

Compatible with breastfeeding but monitor infant for thrush and diarrhoea 
Ciprofloxacin

Metronidazole

Diclofenac

Piroxicam

Ibuprofen

Pentazocine

Diazepam

Lorazepam

Paracetamol

Chloroquine

Sulphadoxine/pyrimethamine

Artesunate

Dexamethasone

Salbutamol

Chlorpheniramine

Promethazine
Avoid if possible until more data is available

Avoid if possible. Animal data suggest it may be carcinogenic

Safety data not documented

Safety data not documented

Compatible with breast feeding

Compatible with breastfeeding in occasional doses. Monitor for apnoea, bradycardia, and cyanosis

Compatible with breastfeeding in single dose Monitor for drowsiness

Compatible with breastfeeding in single dose Monitor for drowsiness

Compatible with breastfeeding

Monitor for haemolysis and jaundice, especially in preterm or infant less than 1 month old

Monitor for jaundice

Compatible with breast feeding

Compatible when used as a single dose. No data on prolonged use

Compatible with breast feeding

Compatible with breastfeeding in single dose Monitor for drowsiness

Compatible with breastfeeding in single dose Monitor for drowsiness

\section{Discussion}

The pattern of medical and gynaecological conditions that afflicted the patients in this study during pregnancy was similar to those reported in South-Eastern Nigeria ${ }^{15}$ and Togo $^{34}$ but varied with those reported in Nepal ${ }^{16}$, South Africa ${ }^{35}$, and Ethiopia $^{36}$. Malaria fever, followed by upper respiratory tract infection, was the most prevalent medical condition in this study. Similar findings have been reported in South-eastern Nigeria ${ }^{15}$ and Togo $^{34}$, but contrasting to emesis gravidarum, heartburn, upper respiratory tract infection, and pain related problems that were reported in other developing16, 35,36 and developed ${ }^{37,38}$ countries.
Malaria in pregnancy is a major public health problem in Nigeria and other malaria endemic countries ${ }^{39}$. The deleterious effects of malaria in pregnancy include maternal anaemia, intrauterine growth restriction, low birth weight, and neonatal mortality ${ }^{39}$. Intermittent use of sulphadoxine/pyrimethamine for malaria prophylaxis in pregnancy has been recommended by the $\mathrm{WHO}^{39}$ and national guidelines of the Federal Republic of Nigeria for malaria treatment $t^{40}$. Unfortunately, less than $10 \%$ of the patients were treated for malaria with chloroquine, sulphadoxine/pyrimethamine, or artesunate; either as a monotherapy or polytherapy, suggesting that antimalarial prophylaxis was rarely prescribed during pregnancy. 
Despite the general concerns over the safety of medicines used during pregnancy, several studies have reported that most pregnant women frequently use more than one medicine over the course of gestation $^{41,42}$. In the present study, after excluding haematinics, $46.1 \%$ women encountered at least one medicine during pregnancy, of which $37.3 \%$ encountered an average of 3 medicines per prescription. The number of medicines encountered per patient in our study was comparable to those encountered $^{1-3}$ in other studies from developing $15,16,35,36$ and developed ${ }^{17,38}$ countries, but differs widely from the number (6.07) reported in Togo ${ }^{34}$. Medical and gynaecological conditions in pregnancy accounted for the high average medicine encountered per patient in this study. The medical conditions would adversely affect the patients and their unborn child, if left untreated with the prescribed medicines ${ }^{7-9}$.

In this study, ATC group B (medicines for blood and blood forming organs) were the most frequently prescribed during pregnancy. Haematinics (iron, vitamin B12, and folic acid) constituted over $90 \%$ of these medicines and consistently remained the most frequently prescribed in similar studies from Nigeria $^{15,26}$ and other developing countries ${ }^{16,34-36}$. Thus, it seems that haematinics were prescribed as prophylaxis against anaemia in pregnancy; a problem that is more common in developing than developed countries $^{43}$. Excluding haematinics, ATC group N (medicines for the nervous system); predominantly analgesics, was the most commonly prescribed, followed by medicines for respiratory system (ATC group R) and anti-infective medicines for systemic use (ATC group J). Similar prescribing pattern has been reported in South-Eastern Nigeria with analgesics, antimalarials, antibiotics, and antiallergens dominating the prescriptions ${ }^{15}$.

Although, a number of similar studies have used various methods to classify medicine risk in pregnancy ${ }^{34,44}$, the US-FDA risk classification system remains the most widely used ${ }^{10,11,15,36,38}$. This system has been criticized for being prone to misinterpretation and misapplication. However, it is undergoing a review with the aim of providing a more clinically useful and informative model ${ }^{45}$. About a half of the types of medicine prescribed to pregnant women in this study belonged to categories A and B which are generally considered safe and are routinely used. However, diclofenac is an exception as its risk increased from category C to D when used in third trimester or near term ${ }^{33}$. The proportion of medicine types (39.1\%) prescribed from category C, and the few $(13 \%)$ prescribed from category $\mathrm{D}$, may not be based just on risk but risk weighed against the benefit. However, it should be recognised that medicines in categories $C$ and $D$ may pose risks similar to a medicine in category $\mathrm{X}^{45}$.

Although, a category $\mathrm{X}$ medicine, which is absolutely contraindicated in pregnancy, was not prescribed in this study, the prescribing of a medicine with unavailable safety data in pregnancy (potassium citrate mixture or mist pot citrate), is however of a great concern. Other studies from Nigeria also have shown that categories $\mathrm{A}$ and $\mathrm{B}$ medicines are prescribed more frequently than category $\mathrm{C}$ or $\mathrm{D}$ medicines, and very rarely were category $\mathrm{X}$ medicines prescribed during pregnancy ${ }^{15,26}$. Our results were however at variance with those reported from other developing and developed countries where a low proportion of women were exposed to category $X$ medicines during pregnancy ${ }^{10,11,36,38}$; thus suggesting that, between countries, there are variations in disease pattern and medicine exposure during pregnancy.

The results of studies of medicine utilization during pregnancy differ widely with the methods, size, and demographic characteristics of the study population ${ }^{36}$. Besides, studies also vary between countries, making comparison between studies and interpretation of results to be difficult. This is further corroborated by the results of the WHO larger study of drug utilization in pregnancy, involving 22 countries, which also highlighted the wide range of disease pattern, treatment approach, medicine exposure, and cultural differences between countries $^{42}$.

Our study evaluated the medicines used during childbirth, which was lacking in the previous studies. Medicines used for genitourinary system (ATC group $\mathrm{G})$ were the most commonly prescribed in labour. Nearly all the patients were administered oxytocin as a first line medicine in the active management of third and fourth stages of labour. However, ergometrine, a second line uterotonic medicine, was administered along with oxytocin to $5.7 \%$ of the patients. This practice is in line with the guidelines of International Federation of Gynaecologists and Obstetricians and International Confederation of Midwives (FIGO-ICM) which is also universally practiced in developing countries $^{46}$. Although ergometrine is contraindicated in patients with an elevated blood pressure, it is still a safe medicine, as well as low dose oxytocin, when used in labour and early pueperium ${ }^{46}$. In America and Europe, systemic medications are the most commonly used form of analgesia in labour, despite being less efficacious than epidural analgesics ${ }^{47}$. In spite of the risks of neonatal respiratory depression, and maternal nausea, vomiting and sedation associated with intrapartum opioids, they remain the most commonly used systemic analgesics in labour ${ }^{48}$. 
The high use of pentazocine for our patients during labour is therefore consistent with the practice in the developed countries ${ }^{47,48}$.

Most medicines used during lactation were prescribed immediately after parturition. Antiinfective medicines (ATC group J); comprising mostly of antibiotics used as a monotherapy or polytherapy, was the most frequently prescribed in this study. This pattern of prescription was comparable to the findings in a similar Danish large study ${ }^{38}$. Excluding caesarean deliveries, antibiotics were prescribed routinely after uneventful normal childbirth as prophylaxis against maternal infectious morbidity and to reduce postpartum endometritis. This practice would however require a strong enough evidence to support routine use of antibiotics after uneventful normal deliveries.

The majority of medicines prescribed during lactation were compatible with breastfeeding; however, quite a number of them would require monitoring of the infants for side effects. Although the proportion of patients who were prescribed furosemide and ciprofloxacin were low, the inhibitory effect of furosemide on lactation and the potential adverse effects of ciprofloxacin on breastfed infants ${ }^{28}$ would require the use of alternative medicines if necessary.

A major strength of our study is using the prescription data from case files of the patients. This provides a more accurate data than interview studies that are characterised by inherent recall bias and under-ascertainment ${ }^{49}$. By systematically auditing the case files of the women during pregnancy, childbirth and pueperium, we were able to perform a holistic evaluation of medicines used in obstetrics; an approach that was lacking in most of the previous studies. Furthermore, our study has provided adequate data on prescription analysis on this subject which is invaluable in the development of guidelines on rational medicine use during pregnancy and maternity. Given that all medicines prescribed were unlikely to be used during pregnancy and lactation, we may have overestimated medicine use in this study. This is however a limitation of this study.

\section{Conclusion}

The study shows considerable medication use during pregnancy, childbirth and lactation. The moderate exposure during pregnancy to medicines with potential harm to the foetus, and further exposure during lactation to breastfed infants, is of great concern. It is suggested that medicine use during pregnancy and lactation should be monitored regularly by analysing prescription data. Prophylactic prescription of antibiotics after uneventful normal childbirth should be guided by evidence-based studies.

\section{References}

1. Schellack G, Schellack N. Pharmacotherapy during pregnancy, childbirth and lactation: principles to consider. S Afr Pharm J 2011; 78: 12-17.

2. Czeizel AE. The estimation of human teratogenic/fetotoxic risk of exposures to drugs on the basis of Hungarian experience: a critical evaluation of clinical and epidemiological models of human teratology. Expert Opin Drug Saf 2009; 8: 283-303.

3. Anderson GD. Pregnancy-induced changes in pharmacokinetics: a mechanistic-based approach. Clin Pharmacokinet 2005; 44: 989-1008.

4. Koren G. Maternal-fetal toxicology: a clinician's guide 2nd ed. New York: Marcel Dekker Inc.; 1994.

5. Kyle PM. Drugs and the foetus. Curr Opin Obstet Gynecol 2006; 18: 93-99.

6. Yankowitz J. Use of medications in pregnancy: general principles, teratology, and current developments. In: Yankowitz J, Niebyl JR, editors. Drug therapy in pregnancy. Baltimore (MD): Lippincott Williams \& Wilkins, 2001.

7. Sachdeva P, Patel BG, Patel BK. Drug use in pregnancy; a point to ponder. Indian J Pharm Sci 2009; 71: 1-7.

8. Hansen WF, Yankowitz J. Pharmacologic therapy for medical disorders during pregnancy. Clin Obstet Gynecol 2002; 45: 136-52.

9. Yankowitz J, Peacock AE, Hansen WF. Safe prescribing practices in pregnancy and lactation. J Midwifery Women's Health 2002; 47: 409-21.

10. Andrade SE, Gurwitz JH, Davis RL, Chan KA, Finkelstein JA, Fortman K, et al. Prescription drug use in pregnancy. Am J Obstet Gynaecol 2004; 191: 398- 407.

11. Gagne JJ, Maio V, Berghella V, Louis DZ, Gonnella JS. Prescription drug use in pregnancy: a population-based study in Regione Emilia-Romagna, Italy. Eur J Clin Pharmacol 2008; 64: 1125-32.

12. Cooper WO, Hernandez-Diaz S, Arbogast PG, et al. Major congenital malformations after first-trimester exposure to ACE inhibitors. N Engl J Med 2006; 54: 2443-51.

13. Chambers CD, Hernandez-Diaz S, Van Marter LJ, et al. Selective serotonin-reuptake inhibitors and risk of persistent pulmonary hypertension of the newborn. N Engl J Med 2006; 354: 576-87.

14. Wyszynski DF, Nambisan M, Surve T, et al. Increased rate of major malformations in offspring exposed to valporate during pregnancy. Neurology 2005; 64: 961-5.

15. Eze UI, Eferakeya AE, Oparah AC, Enato EF. Assessment of prescription profile of pregnant women visiting antenatal clinics. Pharmacy Practice 2007; 5: 135-39.

16. Das B, Sarkar C, Datta A, Bohra S. A study of drug use during pregnancy in a teaching hospital in Western Nepal. Pharmacoepidemiol Drug Saf 2003; 12: 221-25. 
17. Donati S, Baglio G, Spinelli A, Grandolfo ME. Drug use in pregnancy among Italian women. Eur J Clin Pharmacol 2000; 56: $323-28$.

18. Collaborative Group on Drug Use in Pregnancy. An international survey on drug utilization. Int Risk Safety Med 1991; 2: 345-50.

19. Pray WS. Non-prescription products for the pregnant and breast-feeding patient. US Pharm 2007; 32: 10-14.

20. Food and Drug Administration. Final rule for professional labelling of aspirin, buffered aspiring, and aspiring in combination with antacid drug products. Fed Reg 1999; 64 : 49652-55.

21. Buhimschi CS. Endocrinology of lactation. Obstet Gynecol Clin North Am 2004; 31: 963-79.

22. Berlin CM, Paul IM, Vesell ES. Safety issues of maternal drug therapy during breast-feeding. Clin Pharmacol Ther 2009; 85: 20-22.

23. Berlin CM, Briggs GG. Drugs and chemicals in human milk. Semin Fetal Neonatal Med 2005; 10: 149-59.

24. Nice FJ, Snyder JL, Kotansky BC. Breast-feeding and overthe-counter medications. J Hum Lact 2000; 16: 319-31.

25. Lagoy CT, Joshi N, Cragan JD, Rasmussen SA. Medication use during pregnancy and lactation: an urgent call for public health action. JWomen's Health (Larchmt) 2005; 14: 104-9.

26. Gharoro EP, Igbafe AA. Pattern of drug use amongst antenatal patients in Benin City, Nigeria. Med Sci Monit 2000; 6: 84-7.

27. Food and Drug Administration. Requirements on content and format of labelling for human prescription drug and biological products. Federal Register 2006; 71: 3921-97.

28. World Health Organization. Breast feeding and maternal medication: Recommendations for drug in the eleventh WHO Model List of Essential Drugs, 2002. Available at http:/ / whqlibdoc.who.int/hq/2002/55732.pdf (Accessed 31 August, 2011).

29. WHO Collaborating Centre for Drug Statistics Methodology. ACT/DDD Index. Last updated 2010. Available at http:/ /whocc.no/atc_ddd_index/ (Accessed 28 August, 2011).

30. Kelly WJ. Physician's drug handbook, 10th edn. Philadelphia: Lippincott Williams \& Wilkins; 2003.

31. Briggs GC, Freeman RK, Yaffe SJ. A reference guide to foetal and neonatal risk- drugs in pregnancy and lactation, 7th edn. Philadelphia: Lippincott Williams \& Wilkins; 2005.

32. Matsui D, Knoppert D. Drugs and chemicals most commonly used by pregnant women. In: Koren G ed. Medication safety in pregnancy and breastfeeding. New York: McGraw-Hill Professional; 2007. Pp 75-84.

33. Safefetus.com. Pregnancy risk category. Available at http:/ /www.safefetus.com/fda_category.asp (Accessed 28 August, 2011)

34. Potchoo Y, Redah D, Gneni MA, Guissou IP. Prescription drugs among pregnant women in Lome, Togo, West Africa. Eur J Clin Pharmacol 2009; 65: 831-838.

35. Aviv RI, Chubb K, Lindow SW. The prevalence of maternal medication ingestion in the antenatal period. $S$ Afr Med J 1993; 83: 657-60.

36. Kebede B, Gedif T, Gatechew A. Assessment of drug use among pregnant women in Addis Ababa, Ethiopia. Pharmacopidemiol Drug Saf 2009; 18: 462-8.

37. Egen-Lappe V, Hasford J. Drug prescription in pregnancy: analysis of a large statutory sickness fund population. Eur J Clin Pharmacol 2004; 60: 659-66.

38. Olesen C, Steffensen HF, Nielsen GL, de Jong-van den Berg L, Olsen J, Sorensen HT, The Euromap Group. Drug use in first pregnancy and lactation: a population-based survey among Danish women. Eur J Clin Pharmacol 1999; 55: $139-44$.

39. World Health Organization (WHO/AFRO). A strategic frame work for malaria prevention and control during pregnancy in the African region. World Health Organization Regional Office for Africa, Brazzaville-AFR/MAL/04.01; 2004.

40. Federal Ministry of Heath, Nigeria (FMOHN). National guidelines and strategies for malaria prevention and control during pregnancy. Available at http:/ / nmcpnigeria.org/ $\mathrm{f} /$ case-management/mip/National\% 20 Guidelines\% 20 and $\% 20$ Strategies $\% 20$ for $\% 20$ Malaria $\%$ 20Prevention \%20and \%20 Control\% 20During \%20 Pregnancy.pdf (Accessed 31 August, 2011).

41. Bonati M, Bortolus R, Marchetti F, Romer M, Tognoni G. Drug use in pregnancy: an overview of epidemiological (drug utilization) studies. Eur J Clin Pharmacol 1990; 38: 325-8.

42. Collaborative Group on Drug use in Pregnancy (CGDUP). Medication during pregnancy: an intercontinental cooperative study. Int J Obstet Gynecol 1992; 39: 185-96.

43. World Health Organization. The prevalence of anaemia in women: a tabulation of available information. Geneva: Maternal Health and Safe Motherhood Programme, World Health Organization, 1992. 100 p.

44. Rohra DK, Das N, Azam SI, Solangi NA, Memon Z, Shaikh AM, Khan NH. Drug-prescribing patterns during pregnancy in the tertiary care hospitals of Pakistan: a cross sectional study. BMC Preg Childbirth 2008, 8: 24.

45. U.S. Food and Drug Administration. Summary of proposed rule on pregnancy and lactating. Available at http:// www.fda.gov/Drugs/DevelopmentApprovalProcess/ DevelopmentResources/Labelling/ucm093310.htm (Accessed 31 August, 2011).

46. Stanton A, Armbruster D, Knight R, Ariawan I, Gbangbade $\mathrm{S}$, Getachew A, et al. Use of active management of the third stage of labour in seven developing countries. Bull World Health Organ 2009; 87: 207-15.

47. Micheal W. Analgesia in labour: inhalational and parenteral. Anaesth Intensive Care Med 2004; 5: 233-34.

48. El-Wahab N, Robinson N. Analgesia in labour. Obstet Gynaecol Repr Med 2011; 21: 137-41.

49. Rockenbauer M, Olsen J, Czeizel AE, Pedersen L, Sorensen HT, Euromap Group. Recall bias in a case-control surveillance system on the use of medicine during pregnancy. Epidemiology 2001; 12: 461-66. 